\title{
Dynamic Small-Animal PET Imaging of Tumor Proliferation with 3'-Deoxy-3'-18 F- Fluorothymidine in a Genetically Engineered Mouse Model of High-Grade Gliomas
}

\author{
Michelle S. Bradbury ${ }^{1}$, Dolores Hambardzumyan², Pat B. Zanzonico ${ }^{3}$, Jazmin Schwartz ${ }^{3}$, Shangde Cai ${ }^{4}$, Eva M. Burnazi ${ }^{4}$, \\ Valerie Longo ${ }^{5}$, Steven M. Larson ${ }^{1}$, and Eric C. Holland ${ }^{2,6}$ \\ ${ }^{1}$ Department of Radiology, Sloan Kettering Institute for Cancer Research, New York, New York; ${ }^{2}$ Cancer Biology and Genetics, Sloan \\ Kettering Institute for Cancer Research, New York, New York; ${ }^{3}$ Department of Medical Physics, Sloan Kettering Institute for Cancer \\ Research, New York, New York; ${ }^{4}$ Cyclotron and Radiochemistry Core, Sloan Kettering Institute for Cancer Research, New York, New \\ York; ${ }^{5}$ Small-Animal Imaging Core Facility, Sloan Kettering Institute for Cancer Research, New York, New York; and ${ }^{6}$ Neurosurgery \\ Service, Sloan Kettering Institute for Cancer Research, New York, New York
}

$3^{\prime}$-Deoxy-3'-18 F-fluorothymidine ( $\left.{ }^{18} \mathrm{~F}-\mathrm{FLT}\right)$, a partially metabolized thymidine analog, has been used in preclinical and clinical settings for the diagnostic evaluation and therapeutic monitoring of tumor proliferation status. We investigated the use of ${ }^{18} \mathrm{~F}-\mathrm{FLT}$ for detecting and characterizing genetically engineered mouse (GEM) high-grade gliomas and evaluating the pharmacokinetics in GEM gliomas and normal brain tissue. Our goal was to develop a robust and reproducible method of kinetic analysis for the quantitative evaluation of tumor proliferation. Methods: Dynamic ${ }^{18} \mathrm{~F}-\mathrm{FLT}$ PET imaging was performed for $60 \mathrm{~min}$ in gliomabearing mice $(n=10)$ and in non-tumor-bearing control mice $(n=4)$ by use of a dedicated small-animal PET scanner. A 3-compartment, 4-parameter model was used to characterize ${ }^{18} \mathrm{~F}-\mathrm{FLT}$ kinetics in vivo. For compartmental analysis, the arterial input was measured by placing a region of interest over the left ventricular blood pool and was corrected for partial-volume averaging. The ${ }^{18} \mathrm{~F}-\mathrm{FLT}$ "trapping" and tissue flux model parameters were correlated with measured uptake (percentage injected dose per gram [\%ID/g]) values at 60 min. Results: ${ }^{18} \mathrm{~F}-\mathrm{FLT}$ uptake values (\%ID/g) at $1 \mathrm{~h}$ in brain tumors were significantly greater than those in control brains (mean \pm SD: $4.33 \pm 0.58$ and $0.86 \pm 0.22$, respectively; $P<0.0004$ ). Kinetic analyses of the measured timeactivity curves yielded independent, robust estimates of tracer transport and metabolism, with compartmental model-derived time-activity data closely fitting the measured data. Except for tracer transport, statistically significant differences were found between the applicable model parameters for tumors and normal brains. The tracer retention rate constant strongly correlated with measured ${ }^{18} \mathrm{~F}-\mathrm{FLT}$ uptake values $(r=0.85, P<0.0025)$, whereas a more moderate correlation was found between net ${ }^{18} \mathrm{~F}-\mathrm{FLT}$ flux and ${ }^{18} \mathrm{~F}-\mathrm{FLT}$ uptake values $(r=0.61, P<0.02)$. Conclusion: A clinically relevant mouse glioma model was character-

Received Sep. 6, 2007; revision accepted Nov. 20, 2007.

For correspondence or reprints contact: Michelle S. Bradbury, MD, PhD, Department of Radiology, Memorial Sloan Kettering Cancer Center, 1275 York Ave., S-121, New York, NY 10021.

E-mail: bradburm@mskcc.org

COPYRIGHT @ 2008 by the Society of Nuclear Medicine, Inc. ized by both static and dynamic small-animal PET imaging of ${ }^{18} \mathrm{~F}-$ FLT uptake. Time-activity curves were kinetically modeled to distinguish early transport from a subsequent tracer retention phase. Estimated ${ }^{18} \mathrm{~F}$-FLT rate constants correlated positively with $\% \mathrm{ID} / \mathrm{g}$ measurements. Dynamic evaluation of ${ }^{18} \mathrm{~F}-\mathrm{FLT}$ uptake offers a promising approach for noninvasively assessing cellular proliferation in vivo and for quantitatively monitoring new antiproliferation therapies.

Key Words: ${ }^{18} \mathrm{~F}-\mathrm{FLT}$; proliferation; brain tumors

J Nucl Med 2008; 49:422-429

DOI: 10.2967/jnumed.107.047092

$\mathbf{T}$ here is a growing need to develop suitable in vivo imaging strategies for sensitively detecting, characterizing, and monitoring therapeutic responses in malignant brain tumors and for complementing histologic analyses. Serial objective imaging readouts of key metabolic and physiologic processes may improve the diagnostic and therapeutic management of brain tumors by enabling the earlier identification of tumor progression, recurrence, and treatment failure. In patients with gliomas, tumor cell proliferation has been identified as an important surrogate marker of survival, as determined by staining of ex vivo biopsy specimens with proliferation markers, such as Ki67 (1-3). Such in vitro determinations, however, may fail to predict actual therapeutic responsiveness because of biopsy sampling errors, intratumoral variations in pertinent biologic properties, or nonuniform drug delivery (4).

Cell proliferation imaging by PET may address the foregoing limitations, providing a serial 3-dimensional quantitative assessment of tumor growth or regression in vivo. The radiolabeled thymidine analog $3^{\prime}$-deoxy- $3{ }^{\prime}-{ }^{18} \mathrm{~F}$-fluorothymidine $\left({ }^{18} \mathrm{~F}\right.$-FLT) is well suited for this purpose and has 
been used to monitor tumor uptake in models of untreated $(5-7)$ and treated $(8-13)$ preclinical tumors in conjunction with radiation or antiproliferation therapies and, more recently, to characterize both static and kinetic changes in malignant brain tumor uptake in patients (14-20). After its intravenous administration, ${ }^{18} \mathrm{~F}$-FLT is distributed extracellularly and is subsequently transported into the cytosol, where it is phosphorylated by thymidine kinase 1 , an enzyme overexpressed during the DNA synthesis phase of the cell cycle (21). In its phosphorylated form, ${ }^{18} \mathrm{~F}-\mathrm{FLT}$ is metabolically "trapped" in the cell, with essentially no incorporation into DNA. Its limited transport across the intact blood-brain barrier and the absence of proliferating cells in the normal adult brain result in low levels of ${ }^{18} \mathrm{~F}$-FLT within the cranium. In contrast, ${ }^{18} \mathrm{~F}-\mathrm{FDG}$ exhibits high physiologic uptake in the normal brain. Thus, tumor-to-normal brain uptake ratios and tumor specificity are greater for ${ }^{18} \mathrm{~F}$-FLT than for ${ }^{18} \mathrm{~F}$-FDG $(18,22)$.

Brain tumor ${ }^{18} \mathrm{~F}$-FLT uptake measured at various times after injection has been shown to correlate with histopathologic proliferation markers, such as Ki67 or proliferative cell nuclear antigen, in preclinical models and patient studies $(4,11,18)$. In addition, several clinical investigations have assessed uptake in brain tumors dynamically in conjunction with 2-tissue-compartment (4-rate-constant) models $(14,15,19)$, with one such study correlating uptake ratios with rate constants in patients with gliomas (19). Kinetic modeling of dynamic data permits rate constants for the initial transport and subsequent metabolic ("trapping") phases of ${ }^{18} \mathrm{~F}$-FLT uptake to be independently evaluated, the latter rate constant being directly related to the number or rate of proliferating cells within the tumor volume. The ability to characterize these respective components of timeactivity curves may be particularly important in distinguishing changes in tumor proliferation from alterations in ${ }^{18} \mathrm{~F}$-FLT transport, because both factors contribute to overall radiotracer uptake.

In preclinical models, there is a need to implement dynamic imaging and kinetic analysis methods to derive more accurate and specific estimates of functional parameters characterizing tumor metabolism. Unlike recent clinical investigations, only a few preclinical studies have used dynamic imaging for the purpose of determining the time after injection at which maximum tracer uptake occurs. Improving measurements of key biologic parameters that can serve as surrogate markers of tumor responses in clinically relevant mouse models is critical for enabling the future optimization of diagnostic and treatment planning protocols in patients. Along with correlative histologic or serum markers, such methods might enable earlier changes to be made to protocols prescribing conventional therapies or new single or combination pathway-specific inhibitors. Moreover, the application of kinetic analyses to preclinical models may permit relevant tumor biology to be elucidated under conditions that may not be feasible in patients with brain tumors. Such kinetic methods may additionally be applied to the in vivo evaluation and optimization of new diagnostic probes for tumor detection and characterization.

In the present work, we investigated whether ${ }^{18} \mathrm{~F}$-FLT can be used to detect and assess proliferative activity in genetically engineered mouse (GEM) high-grade glioma models that are thought to accurately recapitulate the human disease. We successfully developed and implemented a compartmental modeling approach that permits the discrimination of tracer transport and delivery from tumor proliferation. Compartmental model tracer rate constants were estimated by use of image-derived input and tissue time-activity data (i.e., left ventricular blood clearance and tumor uptake, respectively). To our knowledge, no studies reported to date have dynamically assessed uptake in preclinical brain tumor models with evaluation of the relevant rate constants. We sought to clarify the extent to which tracer transport and metabolism contribute to overall tumor uptake, and we correlated static uptake measurements (percentage injected dose per gram [\% ID/g]) at $1 \mathrm{~h}$ (i.e., a 59- to 60-min time interval) with the metabolic rate constants.

\section{MATERIALS AND METHODS}

\section{Radiopharmaceuticals}

${ }^{18} \mathrm{~F}$-Fluoride in $97 \%$-enriched ${ }^{18} \mathrm{O}$-water was produced through the ${ }^{18} \mathrm{O}(\mathrm{p}, \mathrm{n}){ }^{18} \mathrm{~F}$ nuclear reaction by use of an EBCO cyclotron and target system. ${ }^{18} \mathrm{~F}$-Fluoride ion $(11.1 \mathrm{GBq})$ was trapped on a QMA cartridge and eluted with a mixture of $\mathrm{Bu}_{4} \mathrm{NHCO}_{3}$ and water into vessel 1 , containing $1 \mathrm{~mL}$ of $\mathrm{CH}_{3} \mathrm{CN}$. The solution was vacuum dried with $3.0 \mathrm{~mL}$ of $\mathrm{CH}_{3} \mathrm{CN}$ at $80^{\circ} \mathrm{C}$. The precursor, butoxycarbonyl-dimethoxytrityl-nosyl-lyxothymidine (20 mg; ABX), was dissolved in $0.4 \mathrm{~mL}$ of $\mathrm{CH}_{3} \mathrm{CN}$, and the reaction mixture was heated at $150^{\circ} \mathrm{C}$ for $10 \mathrm{~min}$. The intermediate was passed through a silica Sep-Pak (Waters Associates) and transferred to vessel 2; this step was followed by the addition of ether $(1.5 \mathrm{~mL}, 2$ times) to wash vessel 1 and the silica Sep-Pak. The reaction mixture was dried under vacuum at $80^{\circ} \mathrm{C}$ until the volume was reduced to 0.1 $\mathrm{mL}$. The dimethoxytrityl-protecting group was removed by treatment with $0.4 \mathrm{~mL}$ of $1 \mathrm{~N} \mathrm{HCl}$ for $5 \mathrm{~min}$ at $80^{\circ} \mathrm{C}$. After neutralization with $1.3 \mathrm{~mL}$ of $2 \mathrm{~N}$ sodium acetate, the reaction mixture was purified by use of a Luna $\mathrm{C}_{18}$ column (Phenomenex) and eluted with $8 \%$ ethanol in water. The ${ }^{18} \mathrm{~F}$-FLT peak sample was collected and dried in a rotary evaporator. The final product was dissolved in $3 \%$ ethanol:saline to the desired volume and passed through a $0.22-\mu \mathrm{m}$ sterile filter into a $5.0-\mathrm{mL}$ sterile vial. The radiochemical yield of ${ }^{18}$ F-FLT at the end of synthesis was $20 \%$ decay corrected to the end of bombardment.

\section{Generation of Mouse Brain Tumors}

All animal experiments were done in accordance with protocols approved by the Institutional Animal Care and Use Committee of Memorial Sloan-Kettering Cancer Center and followed National Institutes of Health guidelines for animal welfare. Within $24 \mathrm{~h}$ of birth, Ntv-a/Ink $4 \mathrm{a}^{-/-} \mathrm{Arf}^{-/-}$mice were injected intracranially with $10^{4} \mathrm{DF}-1$ cells infected with and producing RCAS-PDGF retroviral vectors as previously described (23). Mice were monitored daily for symptoms of tumor development. All imaging experiments were performed on mice at 4-6 wk of age. Non-tumor-bearing mice $(n=4)$ served as controls. 


\section{Small-Animal PET Imaging}

Imaging was performed by use of a dedicated small-animal PET scanner (Focus 120 microPET; Siemens Medical Solutions USA, Inc.). Mice were maintained under $2 \%$ isoflurane anesthesia in oxygen at $2 \mathrm{~L} / \mathrm{min}$ during the entire scanning period. One-hour list-mode acquisitions were initiated at the time of injection of 25.9-55.5 MBq $(700-1,500 \mu \mathrm{Ci})$ of ${ }^{18} \mathrm{~F}$-FLT via the lateral tail vein. An energy window of $350-700 \mathrm{keV}$ and a coincidence timing window of $6 \mathrm{~ns}$ were used. The resulting list-mode data were sorted into twelve 5-s (0-1 $\mathrm{min})$ and fifty-nine 60-s (2-60 min) time bins and into 2-dimensional histograms by Fourier rebinning, and transverse images were reconstructed in a $128 \times 128 \times 96$ matrix by filtered backprojection. The image data were corrected for nonuniformity of the scanner response, dead time count losses, and physical decay to the time of injection. No correction was applied for attenuation, scatter, or partial-volume averaging. The measured reconstructed spatial resolution of the Focus 120 scanner is $\sim 1.6 \mathrm{~mm}$ full width at half maximum at the center of the field of view. The counting rates in the reconstructed images were converted to activity concentrations (percentage injected dose per gram of tissue $[\% \mathrm{ID} / \mathrm{g}]$ ) by use of a system calibration factor (0.037 MBq/mL/cps/voxel) derived from the imaging of a mousesize water-equivalent phantom containing ${ }^{18} \mathrm{~F}$.

\section{Small-Animal PET Image Analysis}

Three-dimensional region-of-interest (ROI) analysis of the reconstructed images was performed by use of ASIPro software (Siemens Medical Solutions USA, Inc.) to determine the mean, maximum, and SD of ${ }^{18}$ F-FLT uptake $(\% \mathrm{ID} / \mathrm{g})$ in the tumor, the normal brain, and the left ventricle (LV). In glioma-bearing mice, ROIs were manually placed over the tumor region. Non-tumorbearing mice served as controls for the assessment of ${ }^{18} \mathrm{~F}-\mathrm{FLT}$ uptake, because the small brain size of the mice and the diffuse, infiltrative nature of the tumors limited differentiation of the tumors from normal brain in the same animal. In control animals, volumetric ROI analysis was performed by placing an 8-mminner-diameter ellipsoidal ROI over the brain region and recording the mean, maximum, and SD of the activities (\% ID/g).

\section{Blood Sampling and Generation of Arterial Input Functions}

Given the small size of the mice used for the study, it was not feasible to collect serial blood samples to derive an arterial input function. Blood-pool time-activity curves were obtained by placing an ROI over the LV in the transaxial slice with the largest cross-sectional area of the LV. Several venous samples, taken from the tails of several mice over the course of the study, were assayed for radioactivity to calibrate the image-derived LV input functions.

\section{Compartmental Modeling}

A 3-compartment, 4-rate-constant model, previously validated for somatic tissues (24) and more recently applied to several patient studies $(14,15,19,25)$, was used to describe ${ }^{18} \mathrm{~F}$-FLT kinetics in tumors (Fig. 1). In short, there was an initial transfer of ${ }^{18} \mathrm{~F}-\mathrm{FLT}$ between the arterial blood (or plasma), the first compartment, and an exchangeable tissue space, the second compartment. The third compartment reflected ${ }^{18}$ F-FLT metabolism (retention or trapping) (Fig. 1). The SAAM II program (26-28) was used to implement the compartmental model in Figure 1.

According to this model, $\mathrm{k}(2,1)\left(\right.$ or $\left.\mathrm{K}_{1}\right)$ and $\mathrm{k}(1,2)\left(\right.$ or $\left.\mathrm{k}_{2}\right)$ are rate constants reflecting tracer influx and efflux between the vascular and tissue spaces, respectively, and $\mathrm{k}(3,2)$ ( or $\mathrm{k}_{3}$ ) describes the

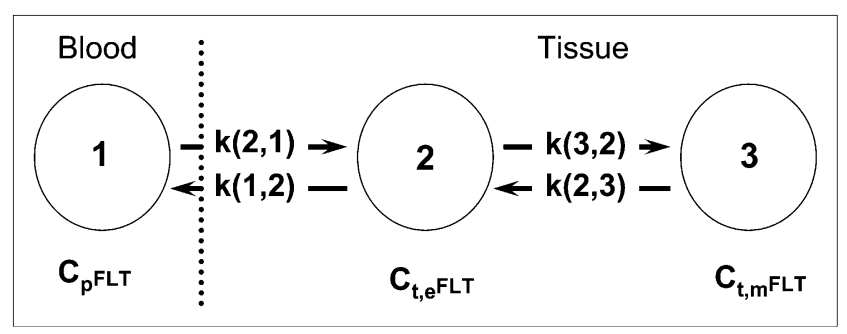

FIGURE 1. Three-compartment, 4-parameter model of ${ }^{18} \mathrm{~F}$ FLT kinetics. ${ }^{18} \mathrm{~F}-\mathrm{FLT}$ is transported from vascular space (1) into tissue, comprising exchangeable compartment (2) and metabolically trapped (i.e., phosphorylated) compartment (3). Four rate constants describe transfer among these compartments. $\mathrm{C}_{\mathrm{p}}^{\mathrm{FLT}}=$ concentration of ${ }^{18} \mathrm{~F}-\mathrm{FLT}$ in arterial plasma; $\mathrm{C}_{\mathrm{t}, \mathrm{e}}{ }^{\mathrm{FLT}}=$ concentration of ${ }^{18} \mathrm{~F}-\mathrm{FLT}$ in exchangeable compartment; $\mathrm{C}_{\mathrm{t}, \mathrm{m}^{\mathrm{FLT}}}=$ concentration of phosphorylated (i.e., trapped) ${ }^{18} \mathrm{~F}-$ FLT.

metabolic retention (phosphorylation) of the tracer (Fig. 1). The potential for reversible tracer trapping is addressed by incorporating the rate constant $\mathrm{k}(2,3)$ (or $\mathrm{k}_{4}$ ), which represents tracer efflux out of the imaging region because of nucleoside transport or dephosphorylation $(29,30)$. Metabolic flux $\left(\mathrm{K}_{\mathrm{FLT}}\right)$ was calculated as follows (24): $\mathrm{K}_{\mathrm{FLT}}=\left(\mathrm{K}_{1} \times \mathrm{k}_{3}\right) /\left(\mathrm{k}_{2}+\mathrm{k}_{3}\right)$.

Because ${ }^{18} \mathrm{~F}$-FLT has been found to be metabolically stable in both the plasma and the urine of tumor-bearing mice over the course of at least $1 \mathrm{~h}$ (11), no correction was made for the accumulation of metabolites in blood over the course of the acquisition. Correction was made for partial-volume averaging of tracer uptake. A fixed value of $5 \%$ was used for the cerebral blood volume (24). Wholeblood and plasma activity concentrations were considered to be equal (24).

\section{Statistical Analysis}

Statistical analyses were performed by use of Excel 2003 (Microsoft) and SigmaPlot (version 8.0; SPSS, Inc.). Differences between tumor-bearing and non-tumor-bearing mice with respect to mean ${ }^{18} \mathrm{~F}-\mathrm{FLT}$ uptake $(\% \mathrm{ID} / \mathrm{g})$ and kinetic parameters were tested for significance by use of unpaired $t$ tests. Average tumor uptake values were correlated with mean $\mathrm{k}_{3}, \mathrm{~K}_{\mathrm{FLT}}$, and $\mathrm{K}_{1}$ values by linear regression analysis. Differences were considered statistically significant at $P<0.05$.

\section{RESULTS}

\section{${ }^{18}$ F-FLT Uptake in Mouse High-Grade Gliomas and Normal Brain Tissue}

The tumor time-activity curves showed a rapid rise and no subsequent (40-60 min) decline in activity. Figure 2 shows representative transverse images of ${ }^{18} \mathrm{~F}$-FLT in normal brain tissue (i.e., non-tumor-bearing animals) (Fig. 2A) and in mouse high-grade gliomas (Fig. 2B) at $1 \mathrm{~h}$ (i.e., a 59to 60 -min time interval) after ${ }^{18} \mathrm{~F}$-FLT injection. Although no appreciable tracer uptake was seen in the brains of the control animals, the high-grade gliomas were clearly seen as an area exhibiting heterogeneous tracer uptake, with a more intense central region presumably reflecting greater proliferative activity. Not unexpectedly, a range of tumor uptake values was observed. Mean $\pm \mathrm{SD}$ and maximum 

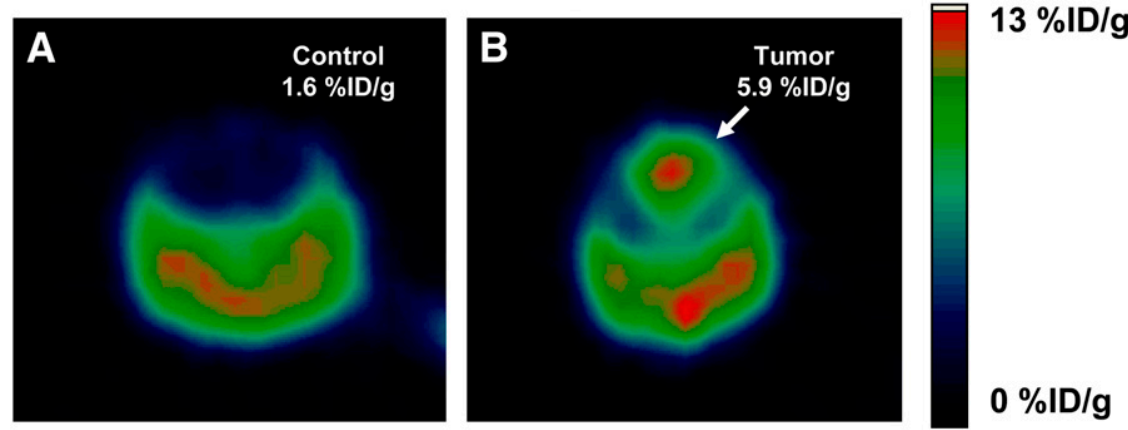

FIGURE 2. Transverse PET images of ${ }^{18} \mathrm{~F}-\mathrm{FLT}$ uptake in non-tumor-bearing (control) (A) and tumor-bearing (B) mouse brains 60 min (i.e., 59-60 min) after intravenous injection. Intense accumulation of radiotracer was seen in GEM highgrade glioma model at level of maximum activity, with no appreciable intracranial uptake being seen in absence of tumor. Mean $\%$ ID/g values are indicated.

$\% \mathrm{ID} / \mathrm{g}$ values for each of the 10 tumor-bearing mice and 4 control mice are shown in Table 1. Mean tumor uptake $(\% \mathrm{ID} / \mathrm{g})$ varied by a factor of 2.6 , ranging from a minimum of $2.32 \pm 0.27$ to a maximum of $5.97 \pm 0.67$, with a group mean of $4.33 \pm 0.58$. Similar differences (nearly a factor of 3 ) were found for the corresponding range of maximum tumor uptake $(\% \mathrm{ID} / \mathrm{g})$. In contrast, substantially lower mean $\% \mathrm{ID} / \mathrm{g}$ values were found in the brains of nontumor-bearing mice, ranging from $0.46 \pm 0.10$ to $1.68 \pm$ 0.38 , with a group mean of $0.86 \pm 0.22$. Differences between control and tumor-bearing mice with respect to mean tumor uptake $(\% \mathrm{ID} / \mathrm{g})$ were statistically significant at $P<$ 0.0004 (Fig. 3A).

\section{Kinetic Analyses of ${ }^{18}$ F-FLT Uptake}

To characterize ${ }^{18} \mathrm{~F}$-FLT uptake kinetics in the spontaneous high-grade glioma model and in normal brain tissue, we generated time-activity curves for tumor-bearing $(n=10)$ and non-tumor-bearing $(n=4)$ cerebral hemispheres. Figure 4 shows differences in the magnitude and rate of ${ }^{18} \mathrm{~F}$-FLT uptake over a 1-h period among representative mice without (Fig. 4A) and with (Figs. 4B and 4C) brain tumors. ${ }^{18}$ F-FLT

TABLE 1

Mean and Maximum ${ }^{18} \mathrm{~F}-\mathrm{FLT}$ Uptake in Spontaneous High-Grade Gliomas and Non-Tumor-Bearing Mice

\begin{tabular}{|c|c|c|c|c|}
\hline \multirow[b]{3}{*}{ Mouse } & \multicolumn{4}{|c|}{ \%ID/g in: } \\
\hline & \multicolumn{2}{|c|}{ Tumor-bearing mice } & \multicolumn{2}{|c|}{ Control mice } \\
\hline & $\overline{\text { Mean } \pm \text { SD }}$ & Maximum & $\overline{\text { Mean } \pm \text { SD }}$ & Maximum \\
\hline 1 & $4.28 \pm 0.66$ & 5.52 & $0.71 \pm 0.21$ & 1.28 \\
\hline 2 & $3.82 \pm 0.60$ & 4.98 & $0.46 \pm 0.10$ & 0.67 \\
\hline 3 & $2.32 \pm 0.27$ & 2.82 & $1.68 \pm 0.38$ & 2.49 \\
\hline 4 & $2.51 \pm 0.45$ & 3.22 & $0.57 \pm 0.20$ & 0.98 \\
\hline 5 & $3.55 \pm 0.42$ & 4.35 & & \\
\hline 6 & $5.96 \pm 0.92$ & 7.60 & & \\
\hline 7 & $5.97 \pm 0.67$ & 7.46 & & \\
\hline 8 & $4.66 \pm 0.50$ & 5.66 & & \\
\hline 9 & $4.30 \pm 0.69$ & 5.42 & & \\
\hline 10 & $5.92 \pm 0.59$ & 7.38 & & \\
\hline Mean $=$ & SD $4.33 \pm 0$. & & $0.86 \pm 0.22$ & \\
\hline
\end{tabular}

${ }^{\star} P<0.0004$ for comparison with control mice (as determined by unpaired $t$ tests). uptake in the representative control brain (Fig. 4A) was $0.71 \pm 0.21 \% \mathrm{ID} / \mathrm{g}$, corresponding to very low (i.e., nearly zero) values for $\mathrm{k}_{3}\left(5.0 \times 10^{-7} / \mathrm{min}\right)$ and $\mathrm{K}_{\mathrm{FLT}}\left(3.65 \times 10^{-7}\right.$ $\mathrm{mL} / \mathrm{min} / \mathrm{g}$ ) (Table 2, control mouse 1). Figure 4D shows the measured time-activity curves and superimposed model fits for the LV (upper curve) and control brain (lower curve). The value for the $\mathrm{k}_{3}$ model parameter in the normal brain was at least 5 orders of magnitude lower than that in the brain tumor, consistent with the suggestion that ${ }^{18} \mathrm{~F}$-FLT does not cross the intact blood-brain barrier to any appreciable degree.

Tumor time-activity curves demonstrated an initial rapid rise in ${ }^{18} \mathrm{~F}$-FLT uptake followed by more gradual uptake and subsequent stabilization of activity during the last 20 min of the study. Peak ${ }^{18}$ F-FLT uptake in tumors was observed at 30-60 min after injection and did not subsequently decline. Progressive increases in proliferative activity in representative brain tumor-bearing mice were reflected in successively higher levels of uptake at $1 \mathrm{~h}$ (Fig. 4B, $2.32 \pm 0.27 \% \mathrm{ID} / \mathrm{g}$; Fig. 4C, $5.92 \pm 0.59 \% \mathrm{ID} / \mathrm{g}$ ). The application of compartmental modeling to the $\mathrm{LV}$ and tissue time-activity curves of one of these tumor-bearing mice (Fig. 4C) generated good agreement between the measured and fitted values (Fig. 4E). The corresponding $\mathrm{k}_{3}$ value was found to be $0.502 \pm 0.002 / \mathrm{min}$ (Table 2, tumor-bearing mouse 10), much higher than the corresponding value in normal brain tissue and reflecting increased tumor cell proliferation.

The mean and range of model parameter estimates for tumor-bearing and control mice are shown in Table 2 and are reasonably consistent with previously published model parameter averages and ranges (24). For all rate constants except $\mathrm{K}_{1}$, the differences between tumor-bearing and control mice were revealed to be statistically significant by unpaired $t$ tests (Table 2 and Fig. 3B); for $\mathrm{K}_{1}$, the values for tumor-bearing and control mice were similar $(0.431 \pm$ 0.012 and $0.458 \pm 0.014$, respectively). This latter finding may be, in part, a reflection of the relatively low bloodtumor barrier permeability measured in these tumor models (Michelle S. Bradbury, unpublished results, August 2007). For all rate constants except $\mathrm{K}_{1}$, maximum and minimum values varied by approximately a factor of 5 ; for $\mathrm{K}_{1}$, larger variations were noted. A statistically significant correlation was found between $\mathrm{k}_{3}$ and 1 -h ${ }^{18} \mathrm{~F}$-FLT uptake in tumor 
FIGURE 3. Static and dynamic measurements of ${ }^{18} \mathrm{~F}-\mathrm{FLT}$ uptake in tumor and control mice in terms of $\% \mathrm{ID} / \mathrm{g}$ (mean $\pm S D)(A)$, kinetic parameters $(B)$, and correlations between $\% \mathrm{ID} / \mathrm{g}$ and retention rate constant, $\mathrm{k}_{3}(\mathrm{C})$, and metabolic flux, $K_{F L T}(D)$. Double asterisks in $A$ indicate statistically significant difference in comparison with results for control mice $(P<0.0004)$. Asterisks in $\mathrm{B}$ indicate mean parameter values of less than $10^{-5}$ and therefore essentially zero. Significantly higher values for $\% \mathrm{ID} / \mathrm{g}$ and all parameters, except for $\mathrm{K}_{1}$, were observed in tumors compared with control mouse brains, and good correlations were observed between $\mathrm{k}_{3}$ or $\mathrm{K}_{\mathrm{FLT}}$ and $\% I D / g .{ }^{\dagger}$ Rate constants are expressed in $\mathrm{mL} / \mathrm{min} / \mathrm{g}$; remaining rate constants are in units of $\min ^{-1}$.

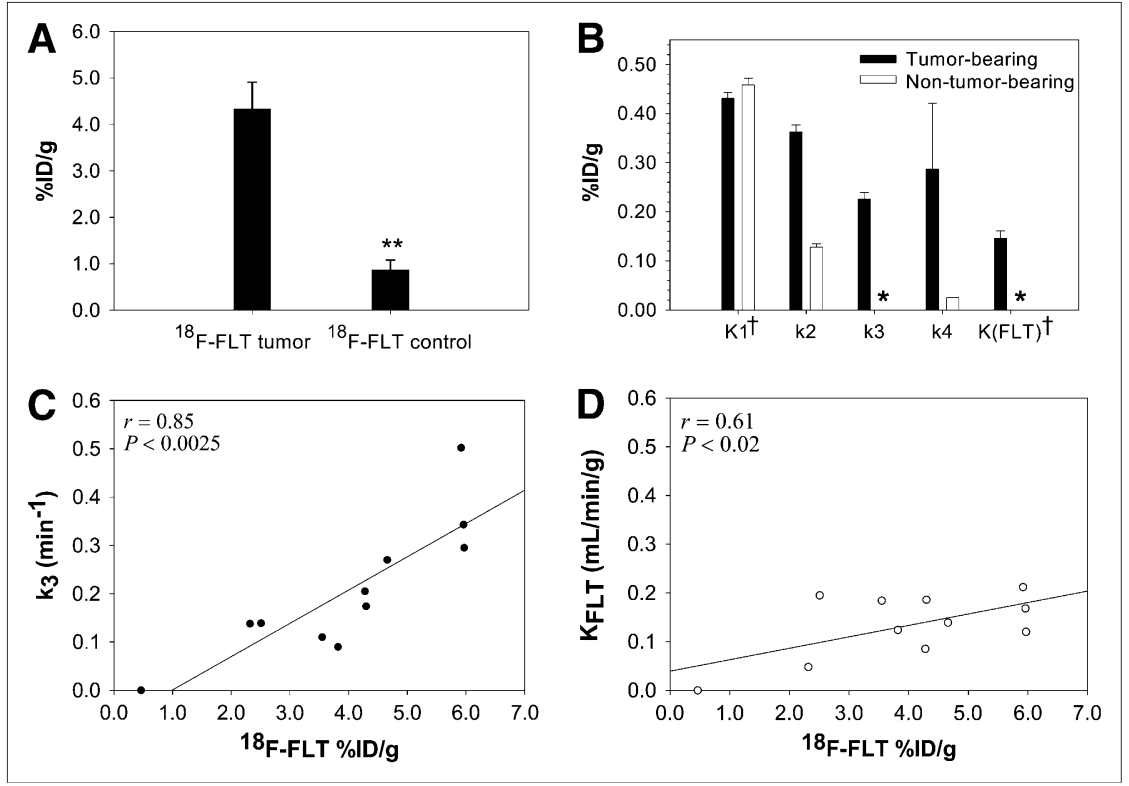

tissue (correlation coefficient $r=0.85, P<0.0025$; Fig. $3 \mathrm{C}$ ); a more moderate correlation was seen between $\mathrm{K}_{\mathrm{FLT}}$ and tumor uptake (correlation coefficient $r=0.61, P<$ 0.02 ; Fig. 3D). Although the early transport of ${ }^{18} \mathrm{~F}-\mathrm{FLT}$ affects tumor uptake, a poorer correlation was found between $\mathrm{K}_{1}$ and 1-h uptake.

Compartmental modeling of non-tumor-bearing mice revealed that the average $K_{1}$ value was significantly larger than all of the other computed rate constants, suggesting that the uptake of ${ }^{18} \mathrm{~F}$-FLT in control mice was dominated by tracer transport. As expected, the $\mathrm{k}_{3}$ and $\mathrm{K}_{\mathrm{FLT}}$ values were equivalent and essentially equal to zero in non-tumorbearing mice but were much larger in glioma-bearing mice, with means of $0.226 \pm 0.013 \mathrm{~min}^{-1}$ and $0.146 \pm 0.015$ $\mathrm{mL} / \mathrm{min} / \mathrm{g}$, respectively.

\section{DISCUSSION}

In the present study, we sought to characterize the kinetics of ${ }^{18}$ F-FLT uptake in a high-grade glioma model with defined genetic alterations that closely recapitulate human disease (31) to develop a practical noninvasive method for assessing cellular proliferation in vivo. Although several clinical studies have modeled the kinetics of ${ }^{18} \mathrm{~F}-\mathrm{FLT}$ uptake in high-grade gliomas by applying compartmental analyses to estimate rate constants $(14,15,19)$, to our knowledge such analyses have not been performed in preclinical brain tumor studies. Implementing such approaches would address the growing need to elucidate key metabolic and physiologic processes in a wide variety of tumor models. Such methods are receiving increasing attention in light of novel probe development, emerging
FIGURE 4. Transverse ${ }^{18} \mathrm{~F}-\mathrm{FLT}$ PET images $(A-C)$ and fitted time-activity curves ( $D$ and $E$ ) corresponding to representative control ( $A$, mouse 1$)$ and tumor-bearing $(C$, mouse 10$)$ mice. Tumor-bearing mice ( $B$ and $C$ ) were selected on basis of minimum (B) and maximum (C) mean \%ID/g values for groups of mice studied; latter values were compared with mean \%ID/g for control mouse (A). Corresponding timeactivity data were kinetically analyzed by compartmental modeling ( $\mathrm{D}$ and $\mathrm{E}$ ); fitted time-activity data are represented by solid or dashed curves, and measured time-activity data are represented by data points.
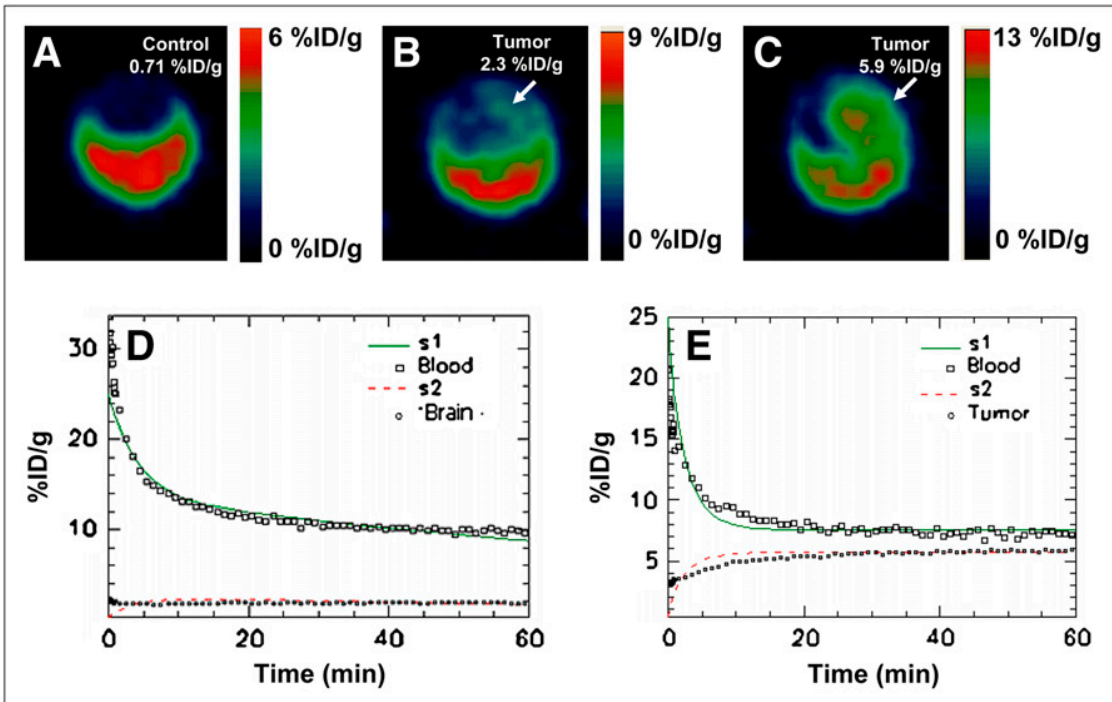
TABLE 2

Kinetic Parameters for High-Grade Gliomas and Non-Tumor-Bearing Mice After ${ }^{18} \mathrm{~F}-\mathrm{FLT}$ Injection, Derived for 3-Compartment Model

\begin{tabular}{|c|c|c|c|c|c|}
\hline \multirow[b]{2}{*}{ Mouse } & \multicolumn{5}{|c|}{ Mean $\pm S D$} \\
\hline & $\begin{array}{c}\mathrm{K}_{1}, \mathrm{~mL} / \mathrm{min} / \mathrm{g} \\
\left({ }^{18} \mathrm{~F}-\mathrm{FLT} \text { transport }\right. \\
\text { from blood } \\
\text { to tissue) }\end{array}$ & $\begin{array}{c}\mathrm{k}_{2}, \min ^{-1} \\
\text { (18 } \mathrm{F}-\mathrm{FLT} \text { efflux } \\
\text { from tissue } \\
\text { to blood) }\end{array}$ & $\begin{array}{c}\mathrm{k}_{3}, \min ^{-1} \\
\left({ }^{18} \mathrm{~F}-\mathrm{FLT}\right. \\
\text { phosphorylation) } \\
\text { (retention) }\end{array}$ & $\begin{array}{c}\mathrm{k}_{4}, \mathrm{~min}^{-1} \\
\left({ }^{18} \mathrm{~F}-\mathrm{FLT} \text { efflux) }\right. \\
\text { (dephosphorylation or } \\
\text { nucleoside transport) }\end{array}$ & $\begin{array}{c}\mathrm{K}_{\mathrm{FLT}}, \\
\mathrm{mL} / \mathrm{min} / \mathrm{g} \\
\left({ }^{18} \mathrm{~F}-\mathrm{FLT}\right. \\
\text { metabolic flux })\end{array}$ \\
\hline \multicolumn{6}{|c|}{ Tumor bearing } \\
\hline 1 & $0.296 \pm 0.003$ & $0.512 \pm 0.006$ & $0.205 \pm 0.007$ & $0.384 \pm 0.010$ & $0.085 \pm 0.003$ \\
\hline 2 & $1.120 \pm 0.030$ & $0.724 \pm 0.021$ & $0.090 \pm 0.020$ & $0.540 \pm 1.240$ & $0.124 \pm 0.029$ \\
\hline 3 & $0.139 \pm 0.004$ & $0.261 \pm 0.059$ & $0.138 \pm 0.008$ & $0.260 \pm 0.011$ & $0.048 \pm 0.005$ \\
\hline 4 & $0.558 \pm 0.023$ & $0.258 \pm 0.001$ & $0.139 \pm 0.013$ & $0.148 \pm 0.017$ & $0.195 \pm 0.022$ \\
\hline 5 & $0.504 \pm 0.025$ & $0.192 \pm 0.011$ & $0.110 \pm 0.016$ & $0.190 \pm 0.024$ & $0.184 \pm 0.031$ \\
\hline 6 & $0.352 \pm 0.005$ & $0.376 \pm 0.009$ & $0.343 \pm 0.010$ & $0.348 \pm 0.010$ & $0.168 \pm 0.008$ \\
\hline 7 & $0.313 \pm 0.009$ & $0.475 \pm 0.015$ & $0.295 \pm 0.014$ & $0.159 \pm 0.007$ & $0.120 \pm 0.009$ \\
\hline 8 & $0.243 \pm 0.001$ & $0.202 \pm 0.001$ & $0.270 \pm 0.027$ & $0.255 \pm 0.002$ & $0.139 \pm 0.019$ \\
\hline 9 & $0.401 \pm 0.013$ & $0.202 \pm 0.006$ & $0.174 \pm 0.014$ & $0.246 \pm 0.020$ & $0.186 \pm 0.019$ \\
\hline 10 & $0.385 \pm 0.008$ & $0.411 \pm 0.009$ & $0.502 \pm 0.002$ & $0.343 \pm 0.014$ & $0.212 \pm 0.006$ \\
\hline Mean $\pm S D$ & $0.431 \pm 0.012$ & $0.363 \pm 0.014$ & $0.226 \pm 0.013$ & $0.287 \pm 0.134$ & $0.146 \pm 0.015$ \\
\hline \multicolumn{6}{|l|}{ Control } \\
\hline 1 & $0.100 \pm 0.003$ & $0.137 \pm 0.004$ & $5.0 \times 10^{-7} \pm 5.0 \times 10^{-10}$ & $0.100 \pm 0.0001$ & $3.65 \times 10^{-7} \pm 3.75 \times 10^{-10}$ \\
\hline 2 & $0.600 \pm 0.004$ & $0.090 \pm 0.006$ & $0 \pm 0$ & $0 \pm 0$ & $0 \pm 0$ \\
\hline 3 & $0.888 \pm 0.040$ & $0.123 \pm 0.006$ & $0 \pm 0$ & $0 \pm 0$ & $0 \pm 0$ \\
\hline 4 & $0.244 \pm 0.009$ & $0.164 \pm 0.011$ & $0 \pm 0$ & $0 \pm 0$ & $0 \pm 0$ \\
\hline Mean $\pm S D$ & $0.458 \pm 0.014$ & $0.128 \pm 0.007$ & $\sim 0$ & $0.025 \pm 00002$ & $\sim 0$ \\
\hline$P^{*}$ & NS & $<0.02$ & $<0.005$ & $<0.001$ & $<0.0002$ \\
\hline
\end{tabular}

${ }^{*}$ For comparison with control mice. NS $=$ not significant.

pharmacologic therapies, and the growing use of spontaneous brain tumor models engineered to express genetic mutations characteristic of human malignancy.

${ }^{18} \mathrm{~F}-\mathrm{FDG}$ has been widely used for imaging glucose transporter expression and hexokinase activity $(32,33)$ and is commonly used for brain tumor diagnosis (34). However, limitations related to its nonspecific uptake in proliferating noncancerous cells or tissues (such as inflammatory cells or granulation tissue) and to the high glucose metabolism of normal brain tissue reduce its detection sensitivity and compromise its ability to monitor the therapeutic response of intracranial lesions $(18,19,22)$. The proliferation marker ${ }^{18} \mathrm{~F}$-FLT is known to be concentrated by a variety of tumors (25,35-37), including those in the central nervous system, and localizes only at very low levels in normal brain tissue. Moreover, because ${ }^{18}$ F-FLT metabolite levels in the blood and urine are known to be very low in mice $(4,11)$, the modeling of ${ }^{18}$ F-FLT PET data can be simplified. This is not the case in humans, in whom the interstitial transport of the glucuronide conjugate of ${ }^{18}$ F-FLT, a blood metabolite, has necessitated the use of a correction factor in compartmental models $(4,14)$.

A limitation in the use of these nucleoside tracers is the significantly higher (on the order of a factor of $10(4,8)$ ) endogenous blood thymidine levels in mice than in humans, with the possibility that thymidine may competitively inhibit thymidine kinase (38) and thereby suppress tissue
${ }^{18}$ F-FLT uptake (4). In one study (7), for example, serum thymidine levels measured at a single time point after the injection of ${ }^{18} \mathrm{~F}$-FLT were found to be highly variable and were weakly correlated with uptake $(\% \mathrm{ID} / \mathrm{g})\left(R^{2}=0.40\right)$. On the other hand, other investigators reported that thymidine levels remained relatively constant in a given species (39). Although not done in the present study, measurement of serum thymidine levels may be warranted in future studies to correct measurements of tissue ${ }^{18} \mathrm{~F}$-FLT uptake.

In the present study, ${ }^{18} \mathrm{~F}$-FLT uptake values at $1 \mathrm{~h}$ after injection were found to be greater in brain tumors than in control mouse brains by a factor of 5, a highly statistically significant difference $(P<0.0004)$. However, in tumor models generally, a static image obtained at an arbitrary time point after injection may not coincide with the time of the maximum uptake or tumor-to-normal tissue contrast. Moreover, static uptake measurements cannot be used to distinguish increased tracer delivery (attributable to increased blood flow or extraction) from increased metabolic trapping and, therefore, may not reliably reflect cellular proliferation. The ability to distinguish between the delivery and retention components of radiotracer uptake may become particularly important in the evaluation of treatment responses. Without information on the relative contributions to overall uptake of the delivery and metabolic trapping of ${ }^{18} \mathrm{~F}-\mathrm{FLT}$, incorrect interpretations regarding tumor proliferation status can result, particularly under conditions in which delivery largely dic- 
tates overall tumor uptake; for example, reduced delivery of ${ }^{18}$ F-FLT to hypoperfused tumors may result in a spuriously low estimate of proliferation status. Because overall tracer uptake is critically affected by tissue perfusion, vascular permeability, and transport into the cell by nucleoside transporters (8), relevant physiologic information may contribute in future studies to further elucidation of the mechanistic basis of alterations in ${ }^{18} \mathrm{~F}$-FLT uptake. Other factors affecting uptake include tumor heterogeneity and size, number of tumor cells in the proliferative phase of the cell cycle at the time of imaging, body temperature variations, the size and placement of ROIs (40), and the quality of the tracer injection.

Kinetic analysis with 3-compartment models has already been applied to ${ }^{18}$ F-FLT time-activity data clinically, with reversible transport of unphosphorylated ${ }^{18} \mathrm{~F}$-FLT between the blood and brain tissue (i.e., an exchangeable tissue compartment) and then a phosphorylation step that leads to ${ }^{18} \mathrm{~F}$-FLT retention (i.e., trapping). Irreversible trapping of phosphorylated ${ }^{18}$ F-FLT, which may lead to a "late" downward curvature of the time-activity curve, has been questioned by some investigators $(15,24)$ and led to the introduction of an efflux rate constant to quantitatively account for this loss. Although the time-activity data in the present study did not exhibit such a loss of ${ }^{18}$ F-FLT activity over the 1-h imaging period, the activity did plateau, and an efflux rate constant $\left(\mathrm{k}_{4}\right)$ was included in the model to optimize its fit to the data. Small and localized differences between the measured and fitted \% ID/g values within the first 10 min after tracer injection were observed in several mice (Fig. 4C) and may be attributable to the increased contribution of noise to the measured time-activity curve during the early tracer equilibration phase, small variations in the blood plasma volume fraction among mice (treated as a fixed parameter in the model), or normal physiologic variations in tissue tracer perfusion, which dominates tracer kinetic behavior early after injection.

\section{CONCLUSION}

The present study demonstrates that dynamic ${ }^{18} \mathrm{~F}$-FLT imaging can characterize tracer kinetics in a clinically relevant, GEM high-grade glioma model and yield estimates of kinetic parameters reflective of tumor cell proliferation. Our preliminary data established the feasibility of using a 3-compartment, 4-parameter model for elucidating critical aspects of tumor biology (such as metabolism or transport). Further studies will investigate whether dynamic measurements of ${ }^{18} \mathrm{~F}$-FLT uptake can serve as surrogates of tumor cell proliferation responses after targeted treatment of GEM high-grade gliomas.

\section{ACKNOWLEDGMENTS}

We thank Yiauchung Sheh for radiopharmaceutical support. Technical services provided by the MSKCC SmallAnimal Imaging Core Facility, supported in part by NIH
Small-Animal Imaging Research Program (SAIRP) grant R24 CA83084, NIH grant 096945, and NIH Center grant P30 CA08748, are also gratefully acknowledged. This work was supported in part by grants from the National Cancer Institute (CA096945 and CA099489) and the Kleberg Foundation.

\section{REFERENCES}

1. Sallinen PK, Haapasalo HK, Visakorpi T, et al. Prognostication of astrocytoma patient survival by Ki-67 (MIB-1), PCNA, and S-phase fraction using archival paraffin-embedded samples. J Pathol. 1994;174:275-282.

2. Reavy-Cantwell JF, Haroun RI, Zahurak M, et al. The prognostic value of tumor markers in patients with glioblastoma multiforme: analysis of 32 patients and review of the literature. J Neurooncol. 2001;55:195-204.

3. Wakimoto H, Aoyagi M, Nakayama T, et al. Prognostic significance of Ki-67 labeling indices obtained using MIB-1 monoclonal antibody in patients with supratentorial astrocytomas. Cancer. 1996;77:373-380.

4. Mankoff DA, Shields AF, Krohn KA. PET imaging of cellular proliferation. Radiol Clin North Am. 2005;43:153-167.

5. Wagner M, Seitz U, Buck A, et al. $3^{\prime}-\left[{ }^{18}\right.$ F $]$ Fluoro- $3^{\prime}$ deoxythymidine $\left\{\left[{ }^{18} \mathrm{~F}\right]\right.$-FLT $\}$ as positron emission tomography tracer for imaging proliferation in a murine B-cell lymphoma model and in the human disease. Cancer Res. 2003;63:2681-2687.

6. Barthel H, Perumal M, Latigo J, et al. The uptake of $3^{\prime}$ deoxy- $3^{\prime}-\left[{ }^{18} \mathrm{~F}\right]$ fluorothymidine into L5178Y tumours in vivo is dependent on thymidine kinase 1 protein levels. Eur J Nucl Med Mol Imaging. 2005;32:257-263.

7. Tseng JR, Dandekar M, Subbarayan M, et al. Reproducibility of $3^{\prime}$-deoxy$3^{\prime}-{ }^{18} \mathrm{~F}$-fluorothymidine microPET studies in tumor xenografts in mice. $\mathrm{J} \mathrm{Nucl}$ Med. 2005;46:1851-1857.

8. Waldherr C, Mellinghoff IK, Tran C, et al. Monitoring antiproliferative responses to kinase inhibitor therapy in mice with $3^{\prime}$-deoxy- $3^{\prime}{ }^{18} \mathrm{~F}$-fluorothymidine PET. J Nucl Med. 2005;46:114-120.

9. Sugiyama M, Sakahara H, Sato K, et al. Evaluation of $3^{\prime}-\operatorname{deoxy}-3^{\prime}-{ }^{18} \mathrm{~F}-$ fluorothymidine for monitoring tumor response to radiotherapy and photodynamic therapy in mice. J Nucl Med. 2004;45:1754-1758.

10. Yang Y-J, Ryu J-S, Kim S-Y, et al. Use of $3{ }^{\prime}$-deoxy- $3^{\prime}-{ }^{18}$ F-fluorothymidine PET to monitor early responses to radiation therapy in murine SCCVII tumors. Eur J Nucl Med Mol Imaging. 2005;33:412-419.

11. Barthel H, Cleij MC, Collingridge DR, et al. $3^{\prime}$-Deoxy- $3^{\prime}-{ }^{18} \mathrm{~F}$-fluorothymidine as a new marker for monitoring tumor response to antiproliferative therapy in vivo with positron emission tomography. Cancer Res. 2003;63:3791-3798.

12. Hsu AR, Cai W, Veeravagu A, et al. Multimodality molecular imaging of glioblastoma growth inhibition with vasculature-targeting fusion toxin VEGF $_{121}$ / rGel. J Nucl Med. 2007;48:445-454.

13. Leyton J, Latigo JR, Perumal M, et al. Early detection of tumor response to chemotherapy by $3^{\prime}$-deoxy- $3^{\prime}-{ }^{18} \mathrm{~F}$-fluorothymidine positron emission tomography: the effect of cisplatin on a fibrosarcoma tumor model in vivo. Cancer Res. 2005;65:4202-4210.

14. Schiepers C, Chen W, Dahlbom M, et al. ${ }^{18}$ F-Fluorothymidine kinetics of malignant brain tumors. Eur J Nucl Med Mol Imaging. 2007;34:1003-1011.

15. Muzi M, Spence AM, O'Sullivan FO, et al. Kinetic analysis of $3^{\prime}$-deoxy-3'-18 Ffluorothymidine in patients with gliomas. J Nucl Med. 2006;47:1612-1621.

16. Buck AK, Bommer M, Stilgenbauer S, et al. Molecular imaging of proliferation in malignant lymphoma. Cancer Res. 2006;66:11055-11061.

17. Saga T, Kawashima H, Araki N, et al. Evaluation of primary brain tumors with FLT-PET: usefulness and limitations. Clin Nucl Med. 2006;31:774-780.

18. Chen W, Cloughesy T, Kamdar N, et al. Imaging proliferation in brain tumors with ${ }^{18}$ F-FLT PET: comparison with ${ }^{18}$ F-FDG. J Nucl Med. 2005;46:945-952.

19. Jacobs AH, Thomas A, Kracht LW, et al. ${ }^{18} \mathrm{~F}$-Fluoro-L-thymidine and ${ }^{11} \mathrm{C}$ methylmethionine as markers of increased transport and proliferation in brain tumors. J Nucl Med. 2005;46:1948-1958.

20. Choi SJ, Kim JS, Kim JH, et al. $\left[{ }^{18} \mathrm{~F}\right] 3^{\prime}$-Deoxy-3'-18 F-fluorothymidine PET for the diagnosis and grading of brain tumors. Eur J Nucl Med Mol Imaging. 2005;32:653-659.

21. Been LB, Suurmeijer AJH, Cobben DCP, et al. ${ }^{18}$ F-FLT-PET in oncology: current status and opportunities. Eur J Nucl Med Mol Imaging. 2004;31:1659-1672.

22. Schaller B. Usefulness of positron emission tomography in diagnosis and treatment follow-up of brain tumors. Neurobiol Dis. 2004;15:437-448.

23. Uhrbom L, Nerio E, Holland EC. Dissecting tumor maintenance requirements using bioluminescence imaging of cell proliferation in a mouse glioma model. Nat Med. 2004;10:1257-1260. 
24. Muzi M, Mankoff DA, Grierson JR, et al. Kinetic modeling of 3'-deoxy-3'fluorothymidine in somatic tumors: mathematical studies. J Nucl Med. 2005;46: 371-380.

25. Muzi M, Vesselle H, Grierson JR, et al. Kinetic analysis of $3^{\prime}$-deoxy-3'fluorothymidine PET studies: validation studies in patients with lung cancer. J Nucl Med. 2005;46:274-282.

26. Barrett PH, Bell BM, Cobelli C, et al. SAAM II: simulation, analysis, and modeling software for tracer and pharmacokinetic studies. Metabolism. 1998; 47:484-492.

27. Berman M, Shahn E, Weiss M. The routine fitting of kinetic data to models: a mathematical formalism for digital computers. Biophys J. 1962;2:275-316.

28. Boston RC, Greif PC, Berman M. Conversational SAAM: an interactive program for kinetic analysis of biological systems. Comput Programs Biomed. 1981;13:111-119.

29. Grierson JR, Schwartz JL, Muzi M, Jordan R, Krohn KA. Metabolism of 3'deoxy-3'-[F-18]fluorothymidine in proliferating A549 cells: validations for positron emission tomography. Nucl Med Biol. 2004;31:829-837.

30. Chishty M, Begley DJ, Abbott NJ, Reichel A. Functional characterisation of nucleoside transport in rat brain endothelial cells. Neuroreport. 2003;14:1087-1090.

31. Hu X, Holland EC. Applications of mouse glioma models in preclinical trials. Mutat Res. 2005;576:54-65.

32. Hatanaka M, Augl C, Gilden RV. Evidence for a functional change in the plasma membrane of murine sarcoma virus-infected mouse embryo cells: transport and transport-associated phosphorylation of ${ }^{14} \mathrm{C}$-2-deoxy-D-glucose. J Biol Chem. 1970;245:714-717.
33. Gallagher BM, Fowler JS, Gutterson JI, et al. Metabolic trapping as a principle of radiopharmaceutical design: some factors responsible for the biodistribution of ${ }^{18} \mathrm{~F}$-2-deoxy-2-fluoro-D-glucose. J Nucl Med. 1978;19:1154-1161.

34. Wong TZ, van der Westhuizen GH, Coleman RE. Positron emission tomography imaging of brain tumors. Neuroimaging Clin N Am. 2002;12:615-626.

35. Kenny LM, Vigushin DM, Al-Nahhas A, et al. Quantification of cellular proliferation in tumor and normal tissues of patients with breast cancer by $\left[{ }^{18} \mathrm{~F}\right]$ fluorothymidine-positron emission tomography imaging: evaluation of analytical methods. Cancer Res. 2005;65:10104-10112.

36. Visvikis D, Francis D, Mulligan R, et al. Comparison of methodologies for the in vivo assessment of ${ }^{18}$ FLT utilisation in colorectal cancer. Eur J Nucl Med Mol Imaging. 2004;31:169-178.

37. Chao KS. Functional imaging for early prediction of response to chemoradiotherapy: $3^{\prime}$-deoxy-3'-fluorothymidine positron emission tomography-a clinical application model of esophageal cancer. Semin Oncol. 2006;33:S59S63.

38. Munch-Petersen B, Cloos L, Tyrsted G, et al. Diverging substrate specificity of pure human thymidine kinases 1 and 2 against antiviral dideoxynucleosides. J Biol Chem. 1991;266:9032-9038.

39. Nottebrock H, Then R. Thymidine concentrations in serum and urine of different animal species and man. Biochem Pharmacol. 1977;26:2175-2179.

40. Krak NC, Boellaard R, Hoekstra OS, et al. Effects of ROI definition and reconstruction method on quantitative outcome and applicability in a response monitoring trial. Eur J Nucl Med Mol Imaging. 2005;32:294-301. 